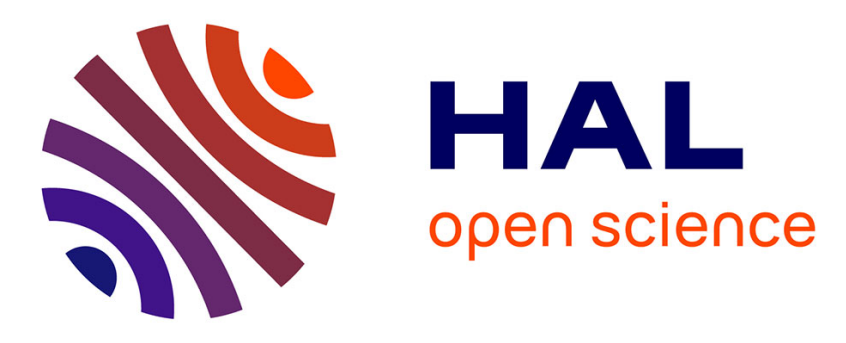

\title{
Indirect excitons in strained GaxIn1-xAs/InP quantum wells
}

\author{
P. Michler, A. Hangleiter, A. Moritz, G. Fuchs, V. Härle, F. Scholz
}

\section{To cite this version:}

P. Michler, A. Hangleiter, A. Moritz, G. Fuchs, V. Härle, et al.. Indirect excitons in strained GaxIn1xAs/InP quantum wells. Journal de Physique IV Proceedings, 1993, 03 (C5), pp.C5-269-C5-272. 10.1051/jp4:1993553 . jpa-00251639

\section{HAL Id: jpa-00251639 https://hal.science/jpa-00251639}

Submitted on 1 Jan 1993

HAL is a multi-disciplinary open access archive for the deposit and dissemination of scientific research documents, whether they are published or not. The documents may come from teaching and research institutions in France or abroad, or from public or private research centers.
L'archive ouverte pluridisciplinaire HAL, est destinée au dépôt et à la diffusion de documents scientifiques de niveau recherche, publiés ou non, émanant des établissements d'enseignement et de recherche français ou étrangers, des laboratoires publics ou privés. 


\title{
Indirect excitons in strained $\mathrm{Ga}_{x} \operatorname{In}_{1-x} \mathrm{As} / \mathrm{InP}$ quantum wells
}

\author{
P. MICHLER, A. HANGLEITER, A. MORITZ, G. FUCHS, V. HÄRLE and F. SCHOLZ
}

4. Physikalisches Institut, Universität Stuttgart, Pfaffenwaldring 57, 70550 Stuttgart, Germany

\begin{abstract}
We present direct experimental evidence that in $G a_{x} \operatorname{In} n_{1-x} A s / \operatorname{In} P$ quantum wells the bandstructure undergoes a direct-to-indirect gap transition in k-space above a critical value of $x$. We observe a drastical increase of the measured radiative exciton lifetimes for samples where $x>x_{c}$, with $\dot{x}_{c}$ depending on well width. Using a six-band $k \cdot p$ calculation of the valence subbands we show that for $x>x_{c}$ the valence band maximum is at $k \neq 0$, i.e. the bandstructure becomes indirect.
\end{abstract}

\section{INTRODUCTION}

Strained-layer quantum wells have recently been subject to considerable interest both from a fundamental point of view and for state-of-the-art applications such as semiconductor lasers $[1,2,3,4]$. The strain introduced by the lattice mismatch primarily leads to a splitting and/or a mixing of the light and heavy hole valence bands and makes the valence subbands in quantum wells much more complex. However, this introduces a new degree of freedom for the valence subbands and makes the effective mass of the valence bands an adjustable parameter [5].

The strained-layer $G a_{x} I n_{1-x} A s / I n P$ material system is particularly interesting since it allows for a variation of strain over a wide range. The strain in the $G a_{x} I n_{1-x} A s$ can be easily controlled for this system as the layer composition is changed from $x=0$ (InAs) to $x=1$ (GaAs). For gallium contents $x<0.47$ the strain is compressive whereas for $x>0.47$ it is tensile.

\section{EXPERIMENTAL}

We performed composition and well width dependent time resolved experiménts on $G a_{x} I n_{1-x} A s /-$ In $P$ quantum wells to investigate the influence of strain on the bandstructure. All of our samples are nominally undoped $G a_{x} I n_{1-x} A s / \operatorname{InP}$ MQW's $(0.45 \leq \mathrm{x} \leq 0.70)$ grown on semi-insulating (100) InP:Fe substrate in a horizontal MOVPE reactor using growth interruptions at all interfaces. The well widths and the well compositions are adjusted by the use of MOVPE growth parameters and are cross-checked by high resolution x-ray diffraction. The MQW's with 4.5- and 6-nm well widths consist of 10 periods of $G a_{x} I n_{1-x} A s$ wells. All wells are separated by $20-\mathrm{nm}$ InP barriers to avoid coupling between the wells. The values of the well widths were kept below the critical layer thickness which is a function of well width and composition $[6,7]$. The time resolved measurements were performed using a photoluminescence setup, where the samples were excited by 5 -ps infrared pulses in the 1.2- 1.6- $\mu \mathrm{m}$ region (for details see ref. [8]).

\section{RESULTS}

The results of the PL decay time measurements at $5 \mathrm{~K}$ are summarized in Fig. 1. The composition dependence of the PL decay time of the samples with 6- and 4.5-nm well width is similar. At low Ga 


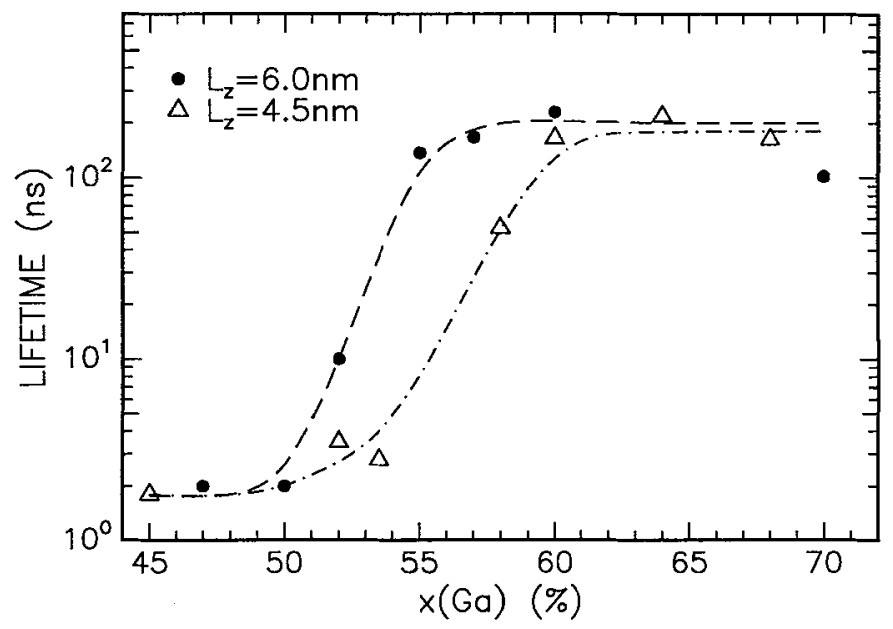

Figure 1: PL lifetime vs gallium composition for the 4.5- and 6- nm MQW's at $5 \mathrm{~K}$. Broken lines are only eye guides

concentrations $x<x_{c}$ we find an almost composition independent lifetime of about 2-3 ns which is in good agreement with the radiative lifetime of an exciton in lattice matched $G a_{0.47} I n_{0.53} A s / \operatorname{In} P$ quantum wells [8]. For gallium concentrations $x>x_{c}$ we observe drastically increased PL decay times by nearly two orders of magnitude. The transition takes place within a narrow region of about 4-5\% around the critical value $x_{c}$. The critical value $x_{c}$ depends on the well width, and is $x_{c} \approx 0.52$ for the samples with $L_{z}=6 \mathrm{~nm}$ and $x_{c} \approx 0.58$ for the samples with $L_{z}=4.5 \mathrm{~nm}$.

By considering the strain dependent change of the valence band structure, the strong increase of the PL decay time can be well understood. We have performed bandstructure calculations using a six band $k \cdot p$ theory based on a Luttinger-Kohn Hamiltonian $[9,10]$. The conduction band offset ratios $\Delta E_{c} / E_{g}$ we used for the calculations were obtained from a linear interpolation between the unstrained case $\Delta E_{c} / E_{g}=0.4$ for $\mathrm{x}=0.47$ and the typ I/ type II transition $\Delta E_{c} / E_{g}=0$ for $\mathrm{x}=$ 0.84 [2]. Fig. 2 shows the calculated valence band structures for a $4.5 \mathrm{~nm}$ quantum well with four different $\mathrm{Ga}$ compositions.

With increasing tensile strain, the light hole subband moves to the valence band ground state (see Fig. 2) which leads to a strong interaction between the light and heavy hole bands. As a consequence a second maximum at $\mathrm{k} \neq 0$ is induced in the highest hole band. For gallium compositions $x>x_{c}$ the second maximum has moved above the maximum at $\mathrm{k}=0$ and the bandstructure becomes indirect. The samples with Ga concentrations below $x_{c}$ have a direct bandstructure and show PL decay times between 2 ns and $3 \mathrm{~ns}$ at low temperatures. The $x \approx x_{c}$ samples, situated close to the transition from direct to indirect bandstructure display decay times between $10 \mathrm{~ns}$ and $100 \mathrm{~ns}$. The samples $x>x_{c}$ situated clearly on the indirect gap side show time constants between $100 \mathrm{~ns}$ and $200 \mathrm{~ns}$.

It is important to note that all samples with a Ga-content $x<x_{c}$ show a single emission line in the PL spectra. This emission line is attributed to excitonic luminescence, since in the examined density region $\left(\approx 1 \cdot 10^{10} \mathrm{~cm}^{-2}\right)$ and at low temperature $(2 \mathrm{~K})$ excitonic recombination dominates the radiative recombination in QW's [8]. In contrast, the spectra for the samples with $x>x_{c}$ are composed of two separate lines. The separation between the two emission lines of $33 \mathrm{meV}$ in all samples corresponds to a LO phonon, revealing the low-energy line as a phonon replica. The interpretation of the low-energy line as a phonon replica is supported by the identical transient decay of the two lines.

For radiative transitions, the recombination in the indirect samples takes place via an indirect exciton, consisting of an electron in the $\Gamma$ conduction band minimum and of a hole in the indirect valence band maximum at $k \neq 0$. In a first approximation, one can describe the expansion of the exciton in $\mathrm{k}$-space with the wave function of the ideal two-dimensional exciton. The inset of Fig. 3 shows the square of the wavefunction $|\Psi(k)|^{2}$ for an exciton in InGaAs in units of the first Brillouin zone $(2 \pi / a)$. The six-band $\mathrm{k} \cdot \mathrm{p}$ calculation yields for the position of the indirect maximum 

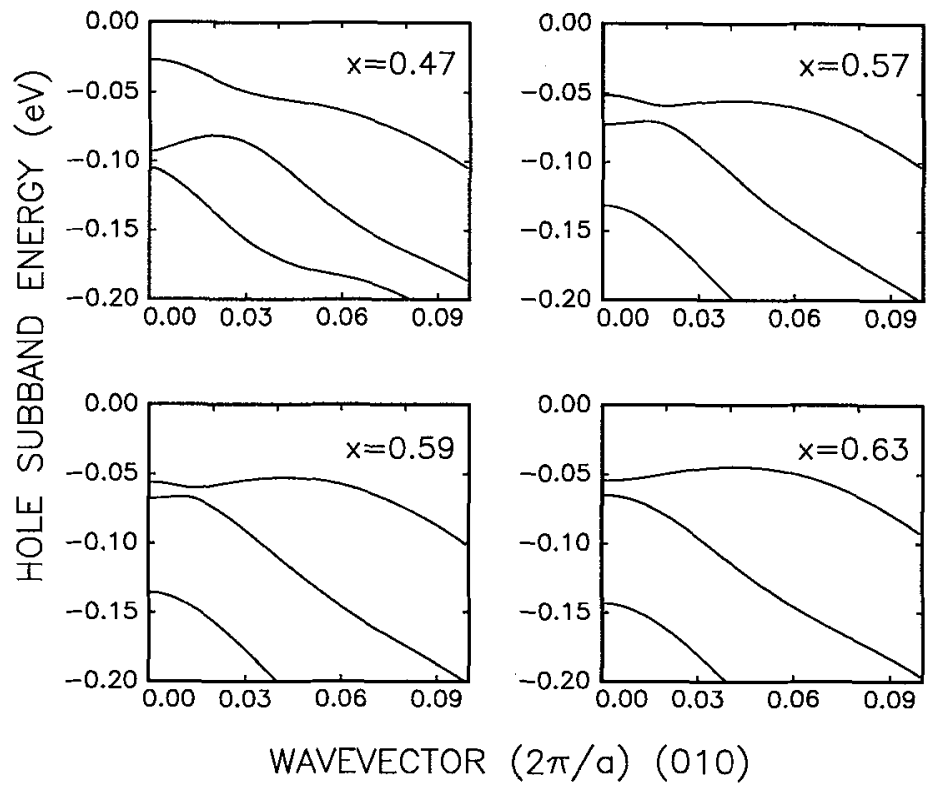

Figure 2: Valence subband structures of $4.5-\mathrm{nm} G a_{x} \operatorname{In} n_{1-x} A s / \operatorname{In} P$ quantum wells with varying contents of gallium calculated by a six band $k \cdot p$ theory.

in the 6 -nm MQW's a value of $k \approx 0.026(2 \pi / a)$. Fig. 3 shows that the square of the wavefunction, which is a measure of the transition probability, is reduced by about two orders in magnitude at $k=0.026(2 \pi / a)$. This means that the transition probability for excitons sitting in the indirect maximum is decreased by two orders in magnitude in comparison to the direct excitons at $\mathrm{k}=0$. This agrees quite good with our lifetime measurements.

In a more precise calculation we consider the occupation probability of the quantum states in the bandstructure and sum over all single probabilities. Fig. 3 shows the results of the lifetime calculations at $5 \mathrm{~K}$ together with the measured values of the 6 -nm samples. The absolute values result from a normalization on the measured values from the samples with a gallium content below the critical value $x_{c}$. If one considers that the theory has no further fitting parameter, the agreement with the experimental values is very good. Nevertheless, the theoretical values are about a factor of two higher than the measured values. One one hand, we observe additional phonon assisted recombination processes which lead to a reduction of the lifetime. But they cannot explain the whole offset between the measured and theoretical lifetimes, since the intensity ratios of the original line to its LO phonon replica is $4: 1$.

One the other hand, an additional broadening of the exciton wave function in k-space would lead to a larger occupation probability at $\mathrm{k}=0$. This would enhance the radiative transition probability and would lead to a better agreement between experimental and theoretical values. The same effect comes from an additional nonradiative recombination path.

The k-value of the indirect maximum in the 4.5-nm MQW is $k=0.026(2 \pi / a)$. Because of the larger $\mathrm{k}$ value of the 4.5-nm MQW's, one expects for the indirect radiative transition a much longer lifetime for the 4.5-nm MQW's, since the square of the exciton wave function at $\mathrm{k}=0$ is much smaller than in the case of the 6 -nm wells. This disagrees with our experimental finding of nearly well width independent lifetimes of 100-200 ns for both, the 6-nm and 4.5-nm samples. Therefore we conclude that the maximum lifetime in the $4.5-\mathrm{nm}$ samples is limited by nonradiative recombination centers. This assumption was supported by our time-integrated measurements. The indirect 4.5-nm samples show a reduced quantum efficiency by a factor of four in comparison to the direct samples, with a further reduction towards high temperatures. The results taken together clearly show that the observed recombination from the indirect maximum in the valence band is partly phonon assisted 


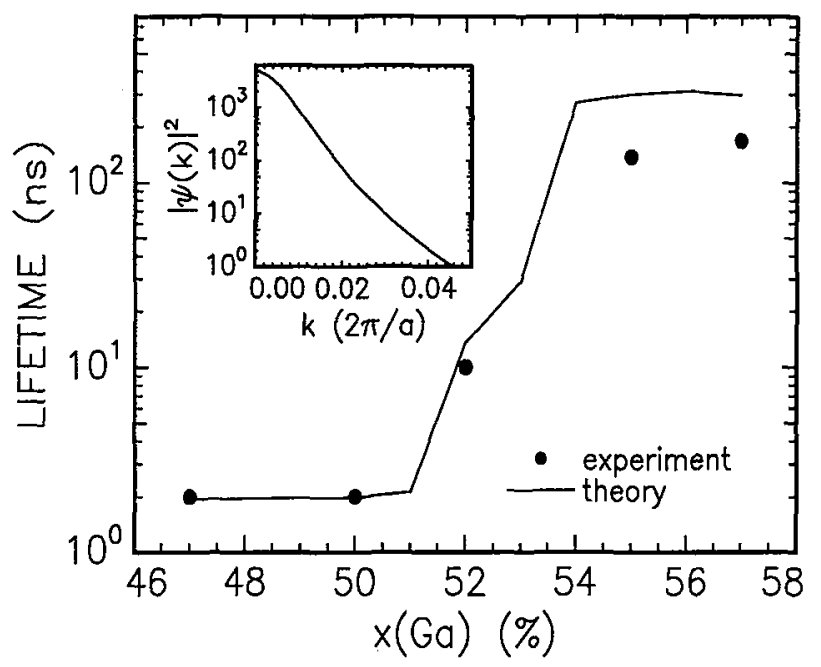

Figure 3: Experimental values of the lifetime and calculated values of the radiative lifetime as a function of gallium content. The inset shows the square of the wave function of the ideal twodimensional exciton in units of the first Brillouin zone.

and the absolute value of the lifetime in the indirect samples is limited by nonradiative recombination centers.

\section{SUMMARY}

In summary we have presented direct experimental evidence that in $G a_{x} I n_{1-x} A s / \operatorname{In} P$ quantum wells the bandstructure undergoes a direct-to-indirect gap transition in $\mathrm{k}$-space above a critical value of $x_{c}$. The experimental results are supported by our six-band $k \cdot p$ calculations of the valence subbands which show that for $x>x_{c}$ the valence band maximum is at $k \neq 0$, i.e. the bandstructure becomes indirect. We observed a drastic increase of the PL lifetime by two orders of magnitude (from 2ns to 100-200 ns) for samples whose gallium content is above the critical value $x_{c}$, with $x_{c}$ depending on well width. The value for $x_{c}$ was determined to be $x_{c} \approx 0.52$ for the $6 \mathrm{~nm}$ well width samples and $x_{c} \approx 0.58$ for the $4.5 \mathrm{~nm}$ well width samples.

[1] T.Y. Wang and G.B. Stringfellow, J.Appl.Phys, 67, 344 (1990).

[2] D. Gershoni, H. Temkin, J.M. Vandenberg, S.N.G. Chu, R.A. Hamm, and M.B. Panish, Phys.Rev.Lett. 60, 448 (1988).

[3] D. Gershoni and H. Temkin, J. Lumin. 44, 381 ( 1989).

[4] S. Murata, K. Naniwae, J. Shimizu, M. Nido, A. Tomita, and A. Suzuki, Electron. Lett. 28, 1456 (1992).

[5] E.P.O' Reilly, Semicond.Sci.Technol. 4, 121 (1989).

[6] R. People and J.C. Bean, Appl.Phys.Lett. 47, 322 (1985).

[7] J.W. Matthews and A.E. Blakeslee, J.Cryst.Growth 27, 118 (1974).

[8] P. Michler, A. Hangleiter, A. Moritz, V. Härle, and F. Scholz, Phys.Rev.B 15, 1671 (1993).

[9] J.M. Luttinger and W. Kohn, Phys.Rev. 97, 869 (1955).

[10] U. Ekenberg, W. Batty, and E.P.O'Reilly, J.Phys.C 5, 533 (1987). 